\title{
Optimization of topical cidofovir penetration using microparticles
}

S. Santoyo, E. Ga de Jalón, P. Ygartua, M.J. Renedo, M.J. Blanco-Príeto*

Department of Pharmacy and Pharmaceutical Technology, University of Navarra, Pamplona 31080, Spain

\section{Corresponding Author}

Telephone + 3400948425600

Fax +3400948425649

e-mail:mjblanco@unav.es 


\section{Abstract}

Cidofovir is a new class of antiviral agent with potent in vitro and in vivo activity against a broad spectrum of herpes viruses. The aim of this work was to obtain a prolonged therapeutic effect of cidofovir in the basal epidermis after its topical application. For this purpose, PLGA microparticles were prepared by solvent evaporation and spray-drying methods. Microparticles prepared by spray-drying showed a encapsulation efficiency of $80 \%$. Conversely, for all the microspheres prepared by the $\mathrm{W} / \mathrm{O} / \mathrm{W}$ solvent evaporation method the encapsulation efficiency was low. Also, microparticles prepared by spray-drying showed a higher burst release. Skin penetration and distribution experiments were carried out with cidofovir-loaded microparticles prepared by spray-drying, since these carriers presented the best characteristics in terms of size and encapsulation efficiency. A cidofovir solution in $0.2 \%$ PVA served for comparison. Penetration experiments were carried out in Franz type diffusion cells with an available diffusion area of $1.76 \mathrm{~cm}^{2}$, using porcine skin. The results obtained showed that the amount of cidofovir penetrated, over a $24 \mathrm{~h}$ time period, was higher with the drug solution than with microparticles. Cidofovir distribution in porcine skin, after topical application of microparticles and drug solution for $24 \mathrm{~h}$, was determined by horizontal slicing of the skin. The profiles obtained for the two formulations showed that the quantity of cidofovir retained in the skin decreased with the depth. Besides the amount of cidofovir found in the basal epidermis $(120-150 \mu \mathrm{m})$ was much higher with microparticles than with the control solution. These data showed that cidofovir-loaded microparticles could improve cidofovir topical therapy since these vehicles increased drug retention in the basal epidermis and decreased its penetration through the skin.

Keywords: cidofovir - Microencapsulation - PLGA - topical application - skin penetration 


\section{Introduction}

Cidofovir ((1-[(S)-3-hydroxy-2-(phosphonomethoxy)propyl] cytosine) (HPMPC)) represents a new class of antiviral agent with potent in vitro and in vivo activity against a broad spectrum of herpes viruses (Bronson et al., 1990). Unlike acyclovir and other nucleoside analogs currently used for clinical therapy of human herpes virus infection, cidofovir does not require virally encoded thymidine kinase for conversion to the active metabolite, cidofovir diphosphate (De Clercq, 1984). Its ability to protect uninfected cells has a great potential in the treatment of recurrent herpes simplex virus infections, HSV-1 and HSV-2, where viral outbreaks lead to secondary cell infection (Spruance and Freeman, 1990). In this way, topical administration of cidofovir has been shown to be effective in the treatment of cutaneous infections of herpes simplex viruses HSV-1 and HSV-2 in animal models (De Clercq and Holy, 1991; Maugdal and De Clercq, 1991; Kern et al., 1995). Besides, acyclovir-resistant HSV-1 in human provided responsive to treatment with topical cidofovir (Snoeck et al., 1993). Consequently, several authors have studied the way to deliver greater amounts of this drug to the target site of virus infections, the basal epidermis, in order to increase the efficacy of the topical therapy. Therefore, the quantification of cidofovir within the skin and after the in vitro percutaneous penetration studies are essential for topical and transdermal research (Touitou et al., 1998).

To minimize systemic exposure and maximize local concentrations, significant effort has been devoted to the development of localized antiviral therapy. It has been shown that poly(lactide-co-glycolide) (PLGA) microparticles penetrate through the stratum corneum and reached the viable epidermis ( $\mathrm{G}^{\mathrm{a}}$ de Jalón et al., 2001a). These particles were used to deliver acyclovir in the basal epidermis ( $\mathrm{G}^{\mathrm{a}}$ de Jalón et al., 2001b). Other authors have also used drug delivery systems for topical delivery of different drugs, such as retinol (Rösseler et al., 1994) 5-fluorouracil-ethyl (Ghorab et al., 1990) and allergenic substances (Brosse et al., 2000). 
In the present study, to obtain a prolonged therapeutic effect of cidofovir in the basal epidermis after its topical application, PLGA microparticles were prepared by solvent evaporation and spray-drying methods.

\section{Materials and methods}

\subsection{Materials}

Cidofovir was a gift from Gilead Sciences (Foster City, CA, USA). Poly(d,l-lactide) (PLA) and Poly(d,l-lactide-co-glycolide) (PLGA) type polymers (PLGA Resomer RG502, RG752, RG756 and PLA R202) were purchased from Boehringer Ingelheim (Ingelheim, Germany). Polyvinyl alcohol (PVA) was obtained from BDH (Poole, England). Dichloromethane (DCM) was from Prolabo (Fontanay, France). Thetrabutylammonium dihydrogenphosphate was supplied by Fluka Chemica (Buchs, Switzerland). Acetonitrile, disodium hydrogenphosphate and other solvents used were of analytical grade and were obtained from Merck (Darmstadt, Germany).

\subsection{Preparation of microspheres by W/O/W solvent evaporation}

Cidofovir was dispersed in $0.5 \mathrm{ml}$ of PVA $1 \%$ and $0.2 \mathrm{~g}$ of polymer was dissolved in 5 $\mathrm{ml}$ of DCM. The suspension and the solution were mixed by ultrasonication under cooling. This so-called inner emulsion was slowly added to $30 \mathrm{ml}$ of a 1\% PVA solution, which was homogenised using an ultraturrax ${ }^{\circledR}$. The resulting W/O/W emulsion was stirred at room temperature for at least three hours to allow solvent evaporation. The microspheres were collected by centrifugation (3000 rpm for $10 \mathrm{~min}$, Biofuge stratus Heraeus Instruments, Hanau, Germany), washed with distilled water and freeze-dried (Blanco-Príeto et al., 1997). 
2.3 Preparation of microspheres by spray-drying

Cidofovir was dispersed in a 5\% (w/w) polymer solution in DCM. The suspension was spray-dryed through a $0.7 \mathrm{~mm}$ nozzle installed in a Mini Spray-Dryer 190 (Büchi, Flawil, Switzerland) (Blanco-Príeto et al., 2000). The product feed was $3 \mathrm{ml} / \mathrm{min}$, inlet and outlet temperatures were at 50 and $40{ }^{\circ} \mathrm{C}$, respectively, aspirator setting at $40 \mathrm{~m}^{3} / \mathrm{h}$, and spray-flow at $450 \mathrm{Nl} / \mathrm{h}$.

\subsection{Microspheres characterization}

The morphology and size of the microspheres were analyzed by light microscopy (Olympus BH-2, Spain) and laser light diffraction (Mastersizer ${ }^{\circledR}$, Malvern, U.K.). The drug content in the microparticles was determined by first dissolving $5 \mathrm{mg}$ of microspheres in $3 \mathrm{ml}$ of DCM followed by extraction with $6 \mathrm{ml}$ of $10^{-4} \mathrm{M} \mathrm{NaOH}$. Cidofovir was assayed in the aqueous phase spectrophotometrically at a wavelength of $274 \mathrm{~nm}$.

\subsection{Burst release}

The drug release after 24 hours (burst release) was determined by dispersing a precisely weighed amount of microspheres in $1.5 \mathrm{ml}$ of isoosmolar PBS (pH 7.4, $67 \mathrm{mM}$ phosphate). The test media was preserved with $0.02 \%(\mathrm{w} / \mathrm{w})$ of sodium azide. Incubation took place in rotating vials at $37^{\circ} \mathrm{C}$. Each assay was replicated three times. The amount of drug released was determined spectrophotometrically.

\subsection{In vitro penetration experiments}

Porcine ears from the local slaughterhouse were cleaned with water and the outer region of the ear was cut. The whole skin was dermatomed (Aesculap ${ }^{\circledR}$ Tuttlingen, Germany) to $1.2 \mathrm{~mm}$ and immediately frozen at $-20^{\circ} \mathrm{C}$. The skin samples were clamped between the two chambers of Franz diffusion cells, with an available diffusion area of $1.76 \mathrm{~cm}^{2}$ (FDC-400, 
Grown Glass Company, Somerville, USA). The stratum corneum faced the donor compartment and the dermis the receptor one.

After stabilisation of the skin, $1.5 \mathrm{~mL}$ of microparticles containing cidofovir (15 mg) or the drug solution (15 mg) were placed, on the skin surface, in the donor compartment. The receptor compartment was filled with $11 \mathrm{~mL}$ of phosphate buffer solution (pH 6), maintained at $37 \pm 1^{\circ} \mathrm{C}$ and stirred at $600 \mathrm{rpm}$ using magnetic stirring bars. $400 \mu \mathrm{L}$ aliquots were collected from the receptor side at designated time intervals, for a $24 \mathrm{~h}$ period, and replaced with the same volume of fresh buffer.

The amount of cidofovir in the receiver phase samples was analysed by highperformance liquid chromatography (HPLC Hewlett-Packard 1050) with ultraviolet detection $(\lambda=274 \mathrm{~nm})$. The column used was a reversed-phase $250 \mathrm{x} 4 \mathrm{~mm} \mathrm{C}_{8}$ LiChrospher Select B (5 $\mu \mathrm{m}$ ) provided by Merck. The mobile phase consisted on 3\% of acetonotrile and $97 \%$ of 1.5 $\mathrm{mM}$ of tetrabutylamonium dihydrogen phosphate and $3.5 \mathrm{mM}$ of disodium hydrogenphosphate adjusted to $\mathrm{pH} 6.0$ with concentrated phosphoric acid. This HPLC method has been previously validated.

\subsection{Skin sectioning and cidofovir extraction}

The amount of the drug retained, in different strata of the skin, was performed by horizontal slicing of the skin in a freeze microtome (2800 Frigocut E, Reichert-Jung, Germany). After permeation experiments, the skin was removed from the diffusion cell and rinsed with distilled water. The treated skin area $\left(1.76 \mathrm{~cm}^{2}\right)$ was frozen in liquid nitrogen and cut with the freeze microtome in order to get horizontal slices of $90 \mu \mathrm{m}$. Cidofovir was extracted from the slices with $300 \mu \mathrm{L}$ of distilled water at $60 \pm 5^{\circ} \mathrm{C}$ for $15 \mathrm{~min}$. Then, the mixture was centrifuged at 8000 rpm (Biofuge Stratus Heraeus Instruments, Germany) and the extract analysed by HPLC. The HPLC conditions were the same described above. 
For the calibration of the extraction procedure, blank samples of skin slices, from different animals and depths, were spiked with three different known amounts of cidofovir solution, and after $3 \mathrm{~h}$ of contact, were extracted as previously described. The extraction recovery was measured by comparing the amount of cidofovir added and extracted. Satisfactory recoveries were obtained from all samples tested ( $\geq 94 \%)$. Cidofovir concentration was expressed as $\mu$ g of cidofovir $/ \mathrm{cm}^{3}$ of skin.

\section{Results and discussion}

\subsection{Microspheres characteristics}

In this study, cidofovir was encapsulated into PLA/PLGA microspheres mainly by the W/O/W solvent evaporation method but also by spray-drying. The nominal amount of cidofovir for all the preparations was $10 \%$, relative to the PLA/PLGA-mass. The mean diameter of the resulted cidofovir-loaded microspheres are listed in table 1. Microspheres prepared by the W/O/W solvent evaporation method using different copolymers showed a mean diameter of 5.2, 5.7, 5.9 and $8.7 \mu \mathrm{m}$ depending on the polymer utilized en their preparation (table 1). The copolymer with the higher molecular weight (RG756) produced organic solution with higher viscosity, resulting in microspheres with the larger diameter (8.7 $\mu \mathrm{m})$. By spray-drying, PLGA particles showed a slightly lower diameter (table 1).

The encapsulation efficiency was significantly higher with spray-drying than with

solvent evaporation (table 1). Actually, particles prepared by spray-drying showed an encapsulation efficiency of up 80\%. Conversely, for all the microspheres prepared by W/O/W solvent evaporation method the encapsulation efficiency was low (table 1). The relative modest encapsulation efficiency achieved can be ascribed to a certain solubility of cidofovir in the organic phase and the leakage of cidofovir to the external aqueous phase where most of the drug was found. Indeed, a key parameter for the successful encapsulation of drugs by this 
method is the insolubility of the drug in the organic polymer solution (Blanco-Príeto et al., 1998).

Besides, it has been shown that increasing the amount of polymer in the organic phase augmented the encapsulation efficiency (Hermann et al., 1995), since raising the amount of polymer increased the viscosity of the primary W/O emulsion. This high viscosity stabilized the internal aqueous phase against coalescence and hence reduced drug loss to the external aqueous phase. In our case, no significant changes in cidofovir encapsulation efficiency was observed when the polymer concentration in the organic phase was augmented (results not shown).

\subsection{Burst release}

The in vitro release of cidofovir from microparticles after 24 hours (burst release) is presented in figure 1. For microparticles prepared by W/O/W solvent evaporation method, the release of cidofovir was independent of the ratio of PLA/PLGA and slightly attenuated when microspheres were prepared with the higher molecular weight copolymer (98000 Da) (figure 1). This results are in agreement with previous studies (Blanco-Príeto et al., 1997).

Microparticles prepared by spray-drying showed a higher burst release than those made by W/O/W solvent evaporation technique (figure 1). This might be explained by the difference in the encapsulation efficiency. The rate of drug release tends to augment with an increased drug content in the particles (Sah et al., 1994; Ertl et al., 1999), due to incrasing amounts of drug located at the suface of the microspheres.

\subsection{Skin penetration and distribution of cidofovir-loaded microparticles}

Since cidofovir-loaded PLGA microparticles prepared by spray-drying showed the best characteristics for topical application (smallest size and the higher encapsulation 
efficiency), these microparticles were used for skin penetration and distribution experiments. Also, in a previous work, it has been shown that PLGA microparticles can penetrate through the stratum corneum and reached the basal epidermis, the target site of the HSV-1 infections ( $\mathrm{G}^{\mathrm{a}}$ de Jalón et al., 2001a).

Porcine skin, specifically the outer region of the ear, was chosen since its use for permeation experiments has been extensively documented (Bathia and Sing, 1998) and it is well suited for representing the human skin permeability (Simon and Maibach, 2000).

In order to study the penetration of the microparticles, $1,5 \mathrm{~mL}$ of the formulation (15 mg of cidofovir) were placed on the skin surface in the donor compartment and $400 \mu \mathrm{L}$ aliquots were collected from the receptor side of the diffusion cell at designated intervals (2, 8, 16 and $24 \mathrm{~h})$. A cidofovir solution in $0.2 \%$ PVA (15 mg) served for comparison. To confirm membrane barrier integrity during the time of the experiment, the skin was observed by light microscopy before and after the permeation experiments, and no skin structural alterations were found.

The penetration profiles of cidofovir from the two formulations, over a $24 \mathrm{~h}$ time period, are shown in figure 2. Cidofovir mean flux values at steady state from microparticles and control solution were $3.63 \pm 0.22$ and $5.27 \pm 0.31 \mu \mathrm{g} / \mathrm{cm}^{2} \mathrm{~h}$ respectively. This result indicated that cidofovir penetration through porcine skin was higher from the drug solution than from microparticles.

At the end of the penetration experiments, cidofovir distribution into the porcine skin, after topical application of microparticles and control solution, during $24 \mathrm{~h}$, was determined by horizontal slicing of the skin. Figure 3 shows the quantity of cidofovir detected, in the different strata of the skin, $24 \mathrm{~h}$ after topical application of microparticles $(15 \mathrm{mg}$ of cidofovir) and the control solution (15 mg of the drug). It has been previously reported that in the porcine skin, the upper $100 \mu \mathrm{m}$ represented mainly the stratum corneum and upper layers 
of viable epidermis, between 100 to $200 \mu \mathrm{m}$ consisted basically of viable epidermis and dermis was located from 200 to $500 \mu \mathrm{m}$ (Jenning et al., 2000a). The distribution profiles obtained for the two formulations showed that the quantity of cidofovir retained in the skin decreased with the depth. The amount of cidofovir found in the first layers of the skin (to 180$270 \mu \mathrm{m}$ of depth) was higher with microparticles than with the control suspension, although from 270 to $800 \mu \mathrm{m}$ there were no significant differences between the two formulations. This result could be explained because microparticles can penetrate through the stratum corneum

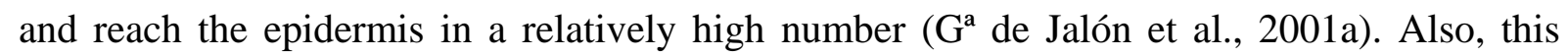
higher cidofovir concentration in the first skin layers could be due to an occlusive effect, since microparticles produced a film on the skin surface, which reduces the transepidermal water loss and favours drug penetration into the skin. The same feature has been observed using acyclovir-loaded microparticles ( $\mathrm{G}^{\mathrm{a}}$ de Jalón et al, 2001b) and solid lipid nanoparticles (Jenning et al., 2000b).

According to the skin morphology of the different slices and the data previously reported, it was assumed that basal epidermis (site of herpes virus lesions) was located between 120 and $150 \mu \mathrm{m}$. Consequently, at the basal epidermis, the quantity of cidofovir found with microparticles was higher than with the solution and this fact pointed out the relevance of these microparticles for cidofovir topical delivery.

\section{Conclusion}

Cidofovir-PLGA microparticles were prepared by spray-drying and by a W/O/W solvent evaporation method. Spray-dried microspheres showed the best characteristics to perform percutaneous penetration studies in terms of size and encapsulation efficiency. Skin penetration and distribution experiments, at $24 \mathrm{~h}$, showed that cidofovir-loaded microparticles increased drug retention in the basal epidermis and decreased its penetration through the skin. 
Consequently, these microparticles could represent a good vehicle to supply the skin with the drug during a prolonged period of time. More experiments are underway to study the percutaneous penetration of cidofovir and cidofovir-loaded microparticles in 48 and 72 hours.

\section{Acknowledgements}

The authors acknowledge the financial support by Gobierno de Navarra (Departamento de Educación), Spain. 


\section{References}

Bhatia, K.S., Singh, J., 1998. Mechanism of transport enhancement of LHRH through porcine epidermis by terpenes and iontophoresis: permeability and lipid extraction studies. Pharm. Res. 15, 1857-1862.

Blanco-Príeto, M.J., Fattal, E., Gulik, A., Dedieu, J. C., Roques, B. P., Couvreur, P., 1997. Characterization and morphological analysis of a cholecystokinin derivative peptideloaded poly(lactide-co-glycolide) microspheres prepared by a water-in-oil-in-water emulsion solvent evaporation method. J. Control. Release 43, 81-87.

Blanco-Príeto, M.J., Fattal, E., Puisieux, F., Couvreur, P., 1998. The multiple emulsion as a common step for the design of polymeric microparticles. In Multiple Emulsions, (Eds.), M. Seiller and J. L. Grossiord, Editions de Santé, Paris (France), pp. 397-435.

Blanco-Príeto, M.J., Besseghir, K., Zerbe, O., Andris, D., orsolini, P., Heimgartner, F., Merkle, H.P., Gander, B., 2000. In vitro and in vivo evaluation of a somatostatin analogue released from PLGA microspheres. J. Control. Release 67, 19-28.

Bronson, J.J., Ferrara, L.M., Hitchcock, M.J.M.; Webb, R.R:, Kern, E.R:, Sokie, K.F.; Martin, J.C., 1990. (S)-1-(3-hydroxy-2-phosphonylmethoxypropyl)cytosine (HPMPC): A potent antiherpesvirus agent. In: Lopez, C. et al (Eds.), Immunobiology and Prophylaxis of Human Herpesvirus Infections, Plenum Press, New York, pp. 277-283.

Brosse, C., Soutif, J.C., Brosse, J.C., 2000. Preparation of microcapsules for skin allergy testing by the solvent evaporation process. J. Microencapsul. 17, 111-116.

De Clercq, E., 1984. Biochemical aspects of the selective antiherpes activity of nucleoside analogues. Biochem. Pharm. 33, 2159-2162.

De Clercq, E., Holy, A., 1991. Efficacy of (S)-1-(3-hydroxy-2phosphonylmethoxypropyl)cytosine in various models of herpes simplex virus infection in mice. Antimicrob. Agents Chemother. 35, 701-706. 
Ertl, B., Platzer, P., Wirth, M., Gabor, F., 1999. Poly(D,L-lactic-co-glycolic acid) microspheres for sustained delivery and stabilization of camptothecin. J. Control. Release 61, 305-317.

Ga de Jalón, E., Blanco-Príeto, M:J; Ygartua, P., Santoyo, S. 2001a., PLGA microparticles: possible vehicles for topical drug delivery. Int. J. Pharm. 226, 181-184.

Ga de Jalón, E., Blanco-Príeto, M:J; Ygartua, P., Santoyo, S., 2001b. Topical application of acyclovir-loaded microparticles: quantification of the drug in porcine skin layers. J. Control. Release 75, 191-197.

Ghorab, M.M., Zia, H., Luzzi, L.A., 1990. Preparation of controlled release anticancer agents. I: 5-Fluorouracil-ethyl cellulose microspheres. J. Microencapsul. 7, 447-454.

Hermann, J., Bodmeier, R., 1995. Somatostatin-containing biodegradable microspheres prepared by a modified solvent evaporation method based on W/O/W-multiple emulsions. Int. J. Pharm. 126, 129-138.

Jenning, V., Schäfer-Korting, M, Gohla, S., 2000a. Vitamin-A-loaded solid lipid nanoparticles for topical use: drug release properties. J. Control. Release 66, 115-126.

Jenning, V.,Gysler, A., Schäfer-Korting, M, Gohla, S., 2000b. Vitamin-A-loaded solid lipid nanoparticles for topical use: occlusive properties and drug targeting to the upper Skin. Eur. J. Pharm. Biopharm., 49, 211-218.

Kern, E.R., PalmerJ., Vogt, P.E., Cundy, K.C., Hitchcock, M.J.M., Sommadossi, J.-P., 1995. Efficacy and pharmacokinetics of topical HPMCP in a genital herpes model. Antiviral Res. 26, 219.

Maugdal, P.L., De Clercq, E., 1991. Effects of phosphonyl-methoxy-alkyl-purine and pyrimidine derivates on TK+ and TK- HSV-1 keratitis in rabbits. Antiviral Res. 16, 93100. 
Rösseler, B., Kreuter, J., Ross, G., 1994. Effect of collagen microparticles on the stability of retinol and its absorption into hairless mouse skin in vitro. Pharmazie 49, 175-179.

Sah, H., Toddywala, R., Chien, Y.W., 1994. The influence of biodegradable microcapsule formulations on the controlled release of a protein. J. Control. Release 30, 201-211.

Simon, G.A., Maibach, H.I., 2000. The pig as an experimental animal model of percutaneous penetration in man: qualitative and quantitative observations. Skin Pharmacol. Appl. Skin Physiol., 13, 229-234.

Snoeck, R., Andrei, G., De Clercq, E., Gerard, M., Clumeck, N., Tricot, G., Sadzot-Delvaux, C., 1993. A new topical treatment for resistant herpes simplex infections. New Engl. J. Med. 329, 968-969.

Spruance, S.L., Freeman, D.J., 1990. Topical treatment of cutaneous herpes simplex virus infections. Antiviral Res. 14, 305-321.

Touitou, E., Meidan, V.M., Horwitz, E., 1998. Methods of quantitative determination of drug localised in the skin. J. Control. Release 56, 7-21. 


\section{Figure legends}

Figure 1. In vitro release of cidofovir from PLGA microspheres in PBS ( $\mathrm{t}=24$ hours). *Spraydryed microparticles

Figure 2. Amount of cidofovir penetrated through porcine skin after $24 \mathrm{~h}$, from microparticles and control solution. Each point represents the mean value \pm S.D. $(n=3)$.

Figure 3. Distribution of cidofovir, in porcine skin layers, following topical administration of microparticles and control solution for 24 h. Each point represents the mean value \pm S.D. $(n=3)$. 
Table 1: Encapsulation efficiencies and size of different microparticles formulations. ${ }^{1} \mathrm{SE}$ : solvent evaporation method; SD: spray-drying

\begin{tabular}{ccccc}
\hline Polymer & Polymer Mw & $\begin{array}{c}\text { Preparation } \\
\text { method }^{1}\end{array}$ & E.E.(\%) & $\begin{array}{c}\text { Mean } \\
\text { diameter }(\mu \mathrm{m})\end{array}$ \\
\hline RG502 & 12000 & SE & $10 \pm 5$ & 5.2 \\
\hline RG502 & 12000 & SD & $80 \pm 10$ & 3.6 \\
\hline RG752 & 16000 & SE & $13.6 \pm 3$ & 5.7 \\
\hline RG756 & 98000 & SE & $14 \pm 3$ & 8.7 \\
\hline R202 & 16000 & SE & $12.1 \pm 5$ & 5.9 \\
\hline
\end{tabular}




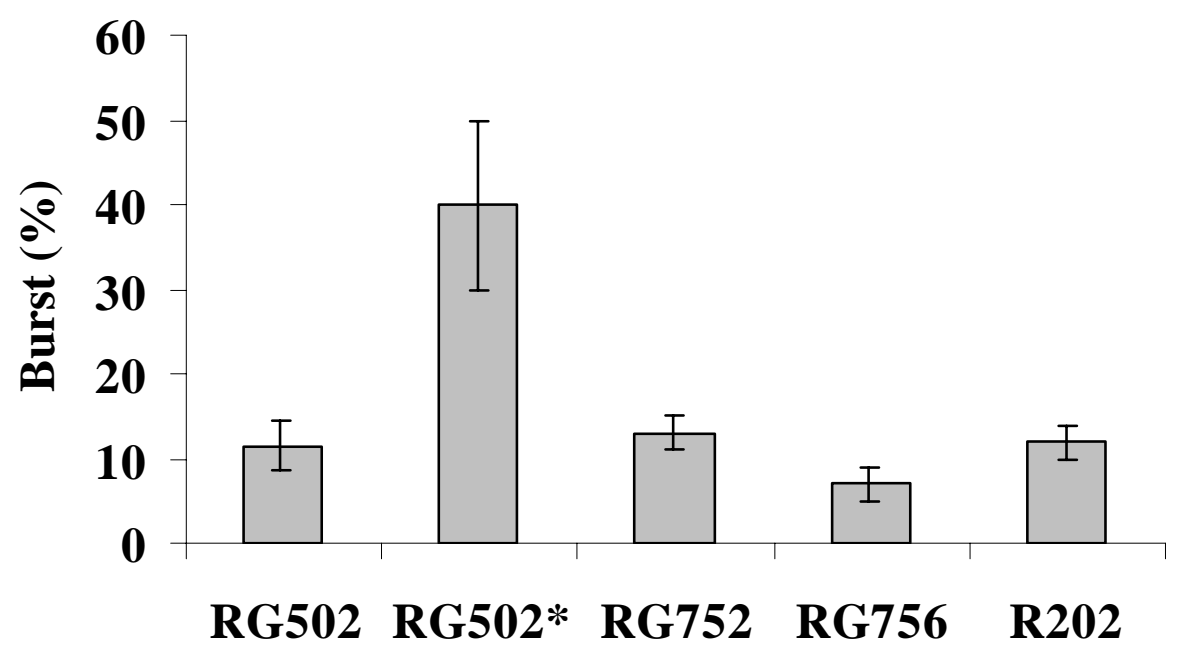




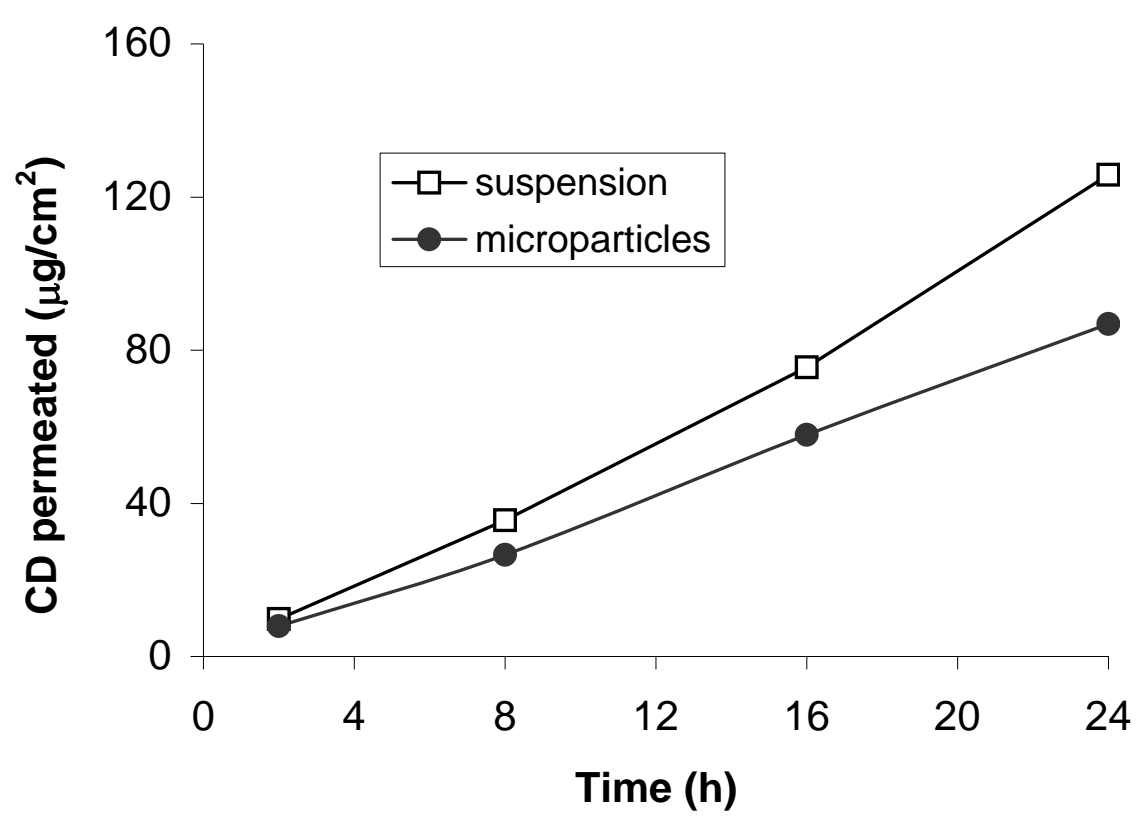




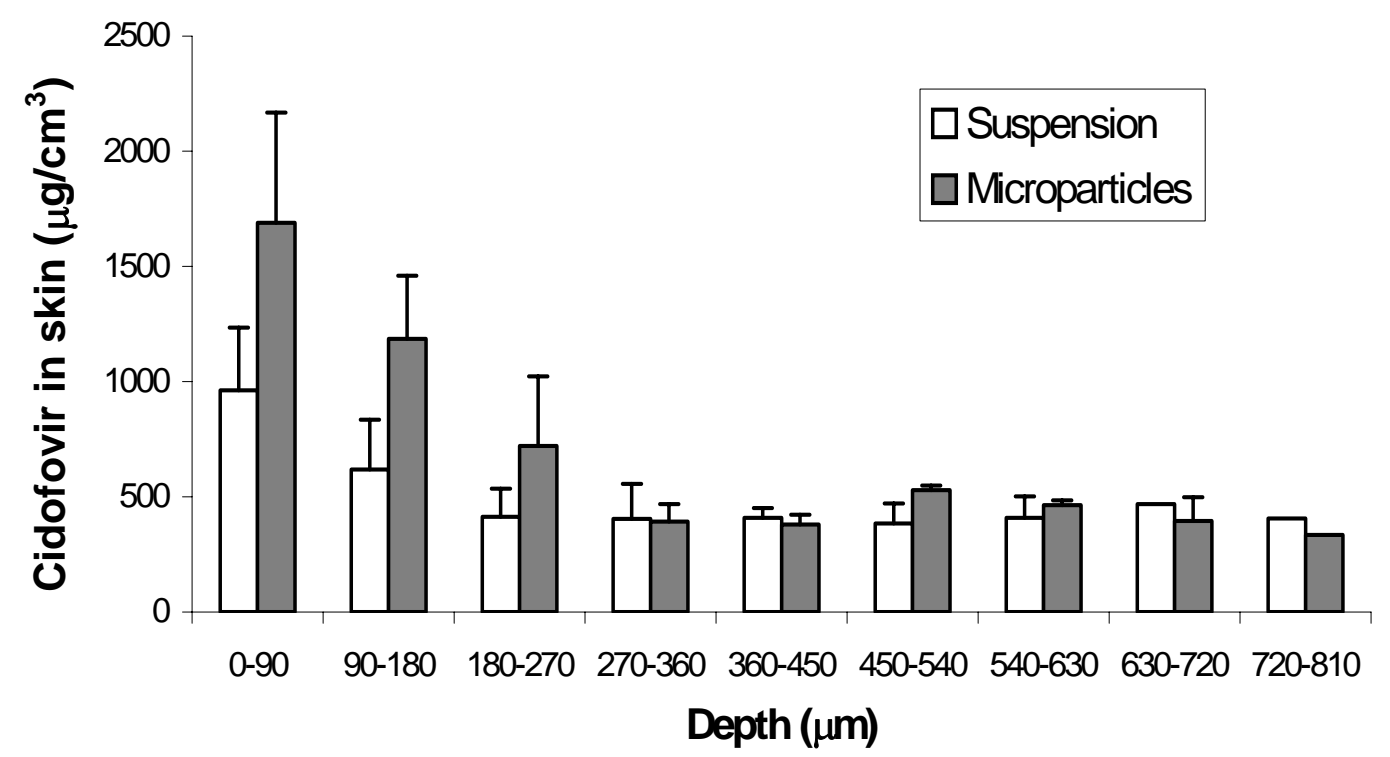

